\title{
The HERO (High-Energy Ray Observatory) simulation
}

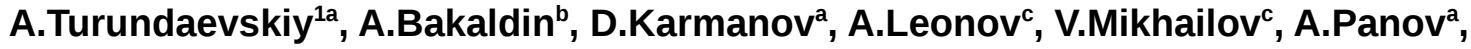 \\ D.Podorozhny ${ }^{\mathrm{a}}$ \\ ${ }^{a}$ Skobetsyn Institute of Nuclear Physics, Moscow State University, Moscow, 119991, Russia \\ ${ }^{b}$ Scientific Research Institute for System Analysis of the Russian Academy of Sciences (SRISA), Moscow, \\ 117218, Russia \\ "National Research Nuclear University "MEPhI", Moscow, 115409, Russia \\ E-mail: turun1966@yandex.ru
}

\begin{abstract}
The main goal of the High-Energy Ray Observatory (HERO) mission is to perform direct measurements of very high energy cosmic ray. Measurements will concern the following scientific goals: detailed study of charge composition of CR in knee region, studies of the energy spectra of Galactic and extragalactic CR, search for signatures of dark matter particles. HERO is planned to be launched onboard a heavy satellite. This experiment is based on the application of a wide aperture $(>2 \pi)$ deep $(\sim 5 \lambda)$ ionization calorimeter. The effective geometrical factor of the apparatus is not less than 8-16 $\mathrm{m}^{2} \mathrm{sr}$ depending on the type of particles. Under the long exposure ( $\sim 10$ years), this mission will make it possible to precisely measure cosmic rays up to $10^{17} \mathrm{eV}$. Wide Monte-Carlo simulations were performed to obtain instrument response for different species of cosmic rays, including charged particles and gammas.
\end{abstract}

35th International Cosmic Ray Conference - ICRC2017

10-20 July, 2017

Bexco, Busan, Korea

1

Speaker 


\section{Introduction}

The central task for understanding of the origin of cosmic rays, their acceleration and propagation in the Galaxy is an interpretation of energy spectrum steepening at $1015-1016 \mathrm{eV}$ (the knee phenomenon). Indirect methods based on expanded atmospheric showers (EAS) registration depend on applied interaction models. It causes significant differences in physical interpretation of obtained data. There are different hypotheses explaining the phenomenon of the knee. Direct measurements of the energy spectra and the chemical composition of cosmic rays are necessary to solve this problem. It is necessary to determine secondary (generated by interaction with interstellar medium) to primary (generated in sources) nuclei ratio at $>100$ $\mathrm{GeV} / \mathrm{n}$ for a study of mechanisms of the cosmic rays propagation in the Galaxy. The cosmic-ray anisotropy is a fundamental problem too. For example, the anisotropy can depend on stochastic character of supernova explosions. The very important problem is investigation of electrons energy spectrum at maximal energies. For high energy electrons the dominant energy loss mechanism is the synchrotron radiation when traversing through the galactic magnetic fields. Therefore, the propagation distance for ultrarelativistic electrons is limited. The high energy electrons spectrum depends on actual nearby sources of cosmic rays. High energy cosmic rays interact with the interstellar gas or the interstellar radiation field, and produce $\gamma$-rays via nucleon-nucleon interactions, electron Bremsstrahlung and inverse Compton scattering. Thus diffusive gamma rays are probe for cosmic rays distribution in the Galaxy. During last decade search of exotic phenomena is performed to determine characteristics of dark matter particles and to find strangelets. The annihilation or decay of hypothetical dark matter par- ticles can cause fine structure in electrons and diffusive gamma energy spectra. Registration of cosmic ray nuclei with anomalous charge to mass ratio should be evidence of strangelets presence.

\section{General requrements}

Direct cosmic rays researches are performed for more than 60 years. During last decade "new generation" experiments based on precision techniques have been performed. Long-duration balloon experiments like ATIC [1], TRACER [2], CREAM [3] allowed to determine fine structure of cosmic rays spectra up to $10^{14} \mathrm{eV}$. With huge interest new data on protons, nuclei and electrons spectra obtained by space experiments PAMELA [4], AMS02 [5], ATIC [6], FermiLAT [7], NUCLEON [8], CALET [9], DAMPE [10] was accepted and interpreted. Above-mentioned experiments won't be able to solve completely these tasks. The main difficulty is insufficient exposition factor for cosmic ray registration for energy range $10^{11}$ - $10^{16} \mathrm{eV}$. The "final” large-scale experiment is necessary. This experiment must be based on application of last achievements of experimental techniques and space technologies.

The total cosmic rays intensity is presented in Table 1. For minimal statistics (100 particles at the end of range) exposition factor must be larger than $100 \mathrm{~m}^{2}$ sr year for a large scale new experiment. 
Table 1.

\begin{tabular}{|l|l|l|l|}
\hline$>\mathrm{E} \quad \mathrm{eV}$ & $10^{14}$ & $10^{15}$ & $10^{16}$ \\
\hline $\mathrm{N}\left(\mathrm{m}^{2}\right.$ sr year $)$ & 2100 & 46 & 0.8 \\
\hline
\end{tabular}

According to tasks the resolution requirements to the experimental device can be determined. The energy resolution must be better than $25 \%$ for nuclei to reconstruct energy spectra and 5\% for electrons and gamma to detect possible spectral peculiarities. The charge resolution must be better than 0.2 charge unit for elemental separation including rare secondary nuclei. The rejection level for electron/proton separation must be better than $10^{4}$ at $10^{11}-10^{12} \mathrm{eV}$ and better than $10^{5}$ at $10^{12}-10^{13} \mathrm{eV}$ to separate electrons from proton background. The rejection level for gamma/electron separation must be better than $10^{2}$ for all energy range.

Now the most universal energy measurements technique is ionisation calorimeter. According to simulation results the above-mentioned parameters can be achieved with thickness larger than 25 radiation lengths and 5 hadronic interaction lengths. The wide aperture $(\sim 2 \pi)$ is necessary to increase geometrical factor. The calorimeter must be homogeneous to register tracks with different directions. The detail cascade registration is necessary for electron/hadrons selection. The additional rejection can be achieved by means of registration of neutrons. Charge measurements can be performed by silicon detectors that were elaborated for accelerator and space experiments. Multi- layer segmented detector system can provide charge resolution and gamma rejection requirements.

The ionization calorimeter is a sufficiently reliable technique but this method is limited significantly by need of heavy absorber to be launched into the orbit. According to the physical conditions the weight of scientific device must be larger than $12 \mathrm{t}$. The long-time exposition of the device is necessary. During all exposition time angle orientation information must be registered. The reliable and continuous power supply and temperature regulation are necessary too. Thus technical characteristics of heavy space vehicles must be taken into account. Heavy class space vehicle designing is necessary. The vehicle characteristics must be close to long-term space station ones except for life support system. The angle orientation determination precision must be better than 1-3 minute. Power supply, temperature regulation, orbit correction system must function more than 10 years.

\section{Experimental techniques optimization}

The HERO calorimeter must consist of 15 identical layers. Every layer is a hexagon (25 $\mathrm{mm}$ thickness, $2025 \mathrm{~mm}$ circumscribed circle diameter). Ionization is registered by polystyrene scintillator ( $1 \mathrm{~g} / \mathrm{cm} 3$ density). Absorber is made of tungsten-copper-nickel alloy (16 g/cm3 density). A layer consists of hexagonal scintillator cells (25 mm inscribed circle diameter). The absorber walls thickness is equal to $5 \mathrm{~mm}$ ( $\sim 1$ radiation length). This structure provide registration of electromagnetic and hadronic showers. The scintillator light signal is registered by the wavelength shifting fibers mounted in three angles $\left(X=0^{\circ}, Y=60^{\circ}, \mathrm{U}=120^{\circ}\right)$, Fig.1-3.

In each direction three fibers are laid in guides.

Each fiber group light signal is registered by one PMT. An opposite end face is polished and covered with a mirror covering. The calorimeter circumscribed circle diameter is equal to 
$2025 \mathrm{~mm}$, height is equal to $455 \mathrm{~mm}$. The mass is equal to $10 \mathrm{t}$. Number of information channels is equal to 3060 .

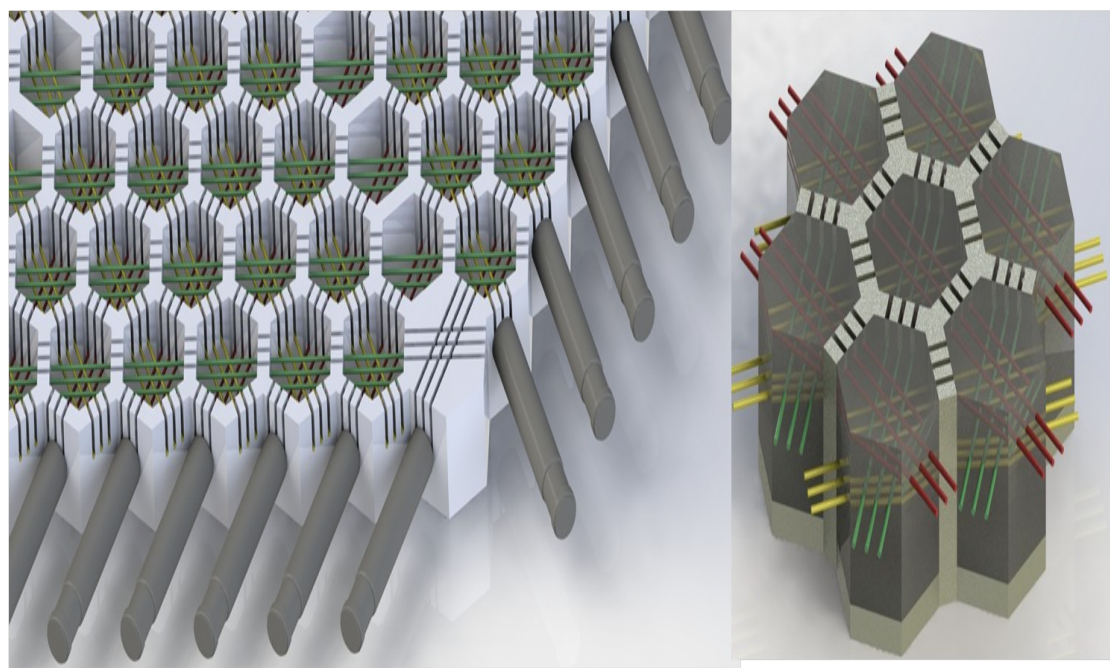

Figure 1: detectors layout .
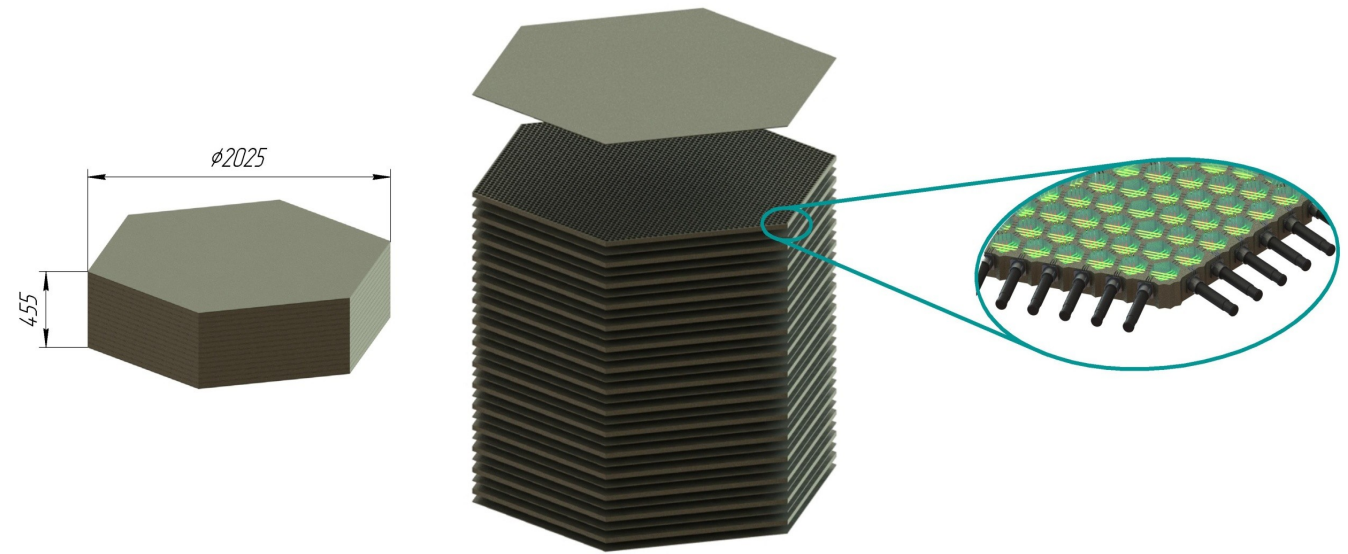

Figure 2: calorimeter

The calorimeter scheme is showed in Fig.4. The calorimeter is surrounded with silicon four-layer charge measurement system with overlapping not less than $95 \%$ of the area. Charge measurement system consists of independent ladders. Each ladder is mounted on planes and consists of 3-16 detectors (100x100x0.5 mm each one). Every detector is divided into 100 independent pads. There are $\sim 2000$ detectors in charge measurement system (i.e. $\sim 2 \times 10^{5}$ independent channels). The calorimeter and charge measurement system are placed inside the cylindrical vessel, truncated on the bottom side of a calorimeter, and established on a bearing platform. The scheme of charge measurement system and the vessel is presented in Fig.3

Additional improvement of rejection can be achieved by neutrons registration. Number of neutrons generated by electromagnetic shower is significantly less than for hadronic shower with the same energy. For example neutron yield distribution is presented in Fig.5 for electron and proton at $\mathrm{E}=10^{11} \mathrm{eV}$. Fig.6. To optimize scientific apparatus neutrons can be registered by 
the same detectors as ionization losses. Scintillator includes gadolinium ( 0.3\%). Ionization and neutron signals are distinguished by time delay. Most of neutrons are termalized and captured by Gd or escaped from spectrometer for $200 \mu$ s. Neutrons registration improves rejection level and additionally at high energies $\left(>10^{14} \mathrm{eV}\right)$ neutrons can be used to determine primary energy.

The optimal orientation is by a lateral side to the Earth. For this variant geometrical factor is about $50 \%$ larger than for parallel orientation.

The Monte-Carlo s of charge measurements was performed to determine charge errors.

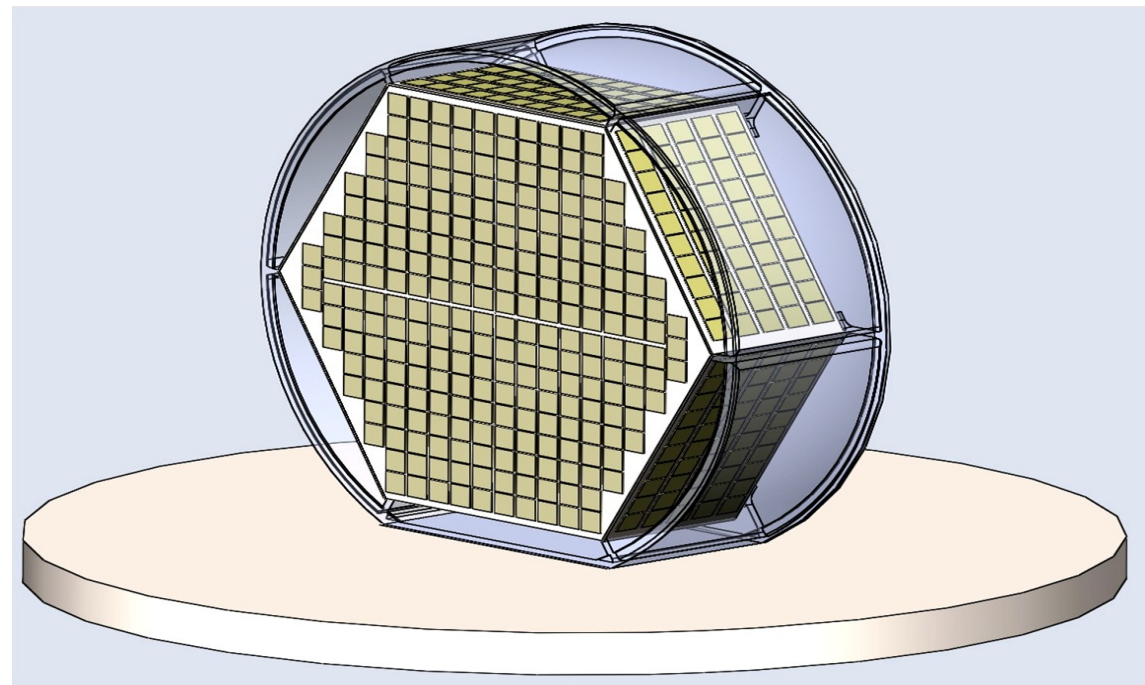

Figure 3: Schematical model of the instrument.

\section{The HERO Monte-Carlo simulation}

Wide Monte-Carlo simulations were performed to obtain instrument response for different species of cosmic rays, including charged particles and gammas. The goal of this work is to improve physical characteristics of the instrument to reach better the angular and energy resolutions than current space missions and ground-based experiments. GEANT Monte-Carlo model of the instrument is shown in figure 4. It is based on GEANT 4.10.03 patch01 simulation package (released 24 February 2017) with hadron model : QGSP_BERT_HP. HP (high precision neutron) model for neutron elastic, inelastic, capture and fission below $20 \mathrm{MeV}$, allow precise transportation of neutrons. Examples of simulated events are shown in figures 5-6. Along with the instrument simulation particles tracing in magnetosphere were performed to obtain detector response at different orbit positions and orientations. Figure 7 shows positron track in magnetosphere and figure 8 demonstrates detector angular distributions of electrons and positrons .Due to large instrument acceptance the method can be used for particle identification up to $100 \mathrm{GeV}$. 


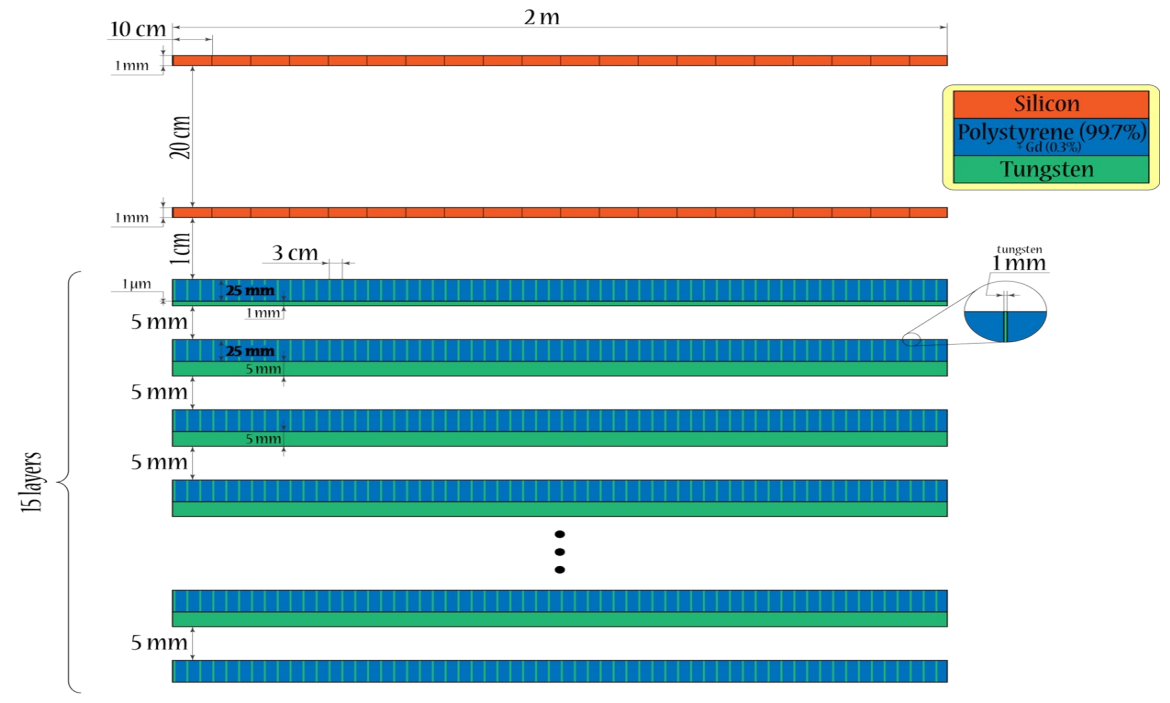

Figure 4: Monte-Carlo model of the instrument.

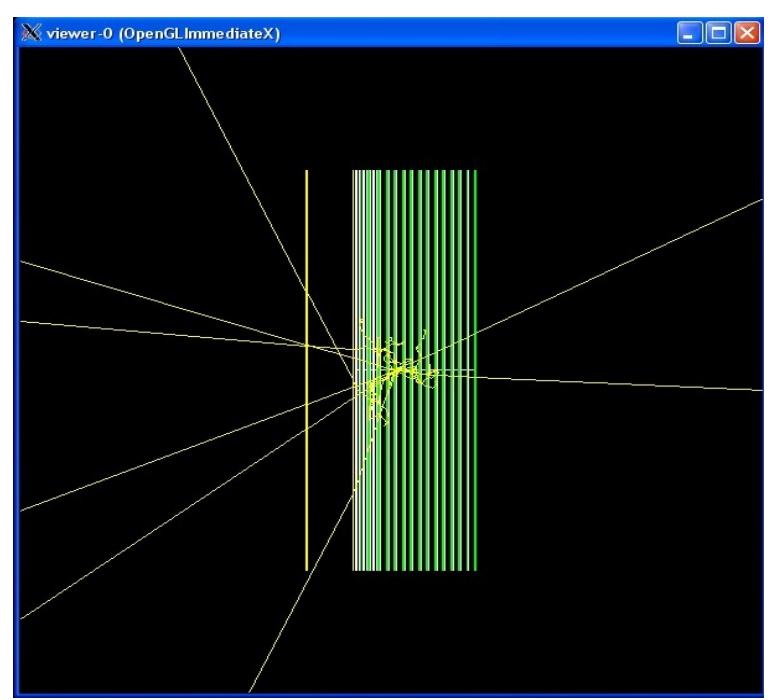

Figure 5: Simulation of neutron tracks from a proton interaction
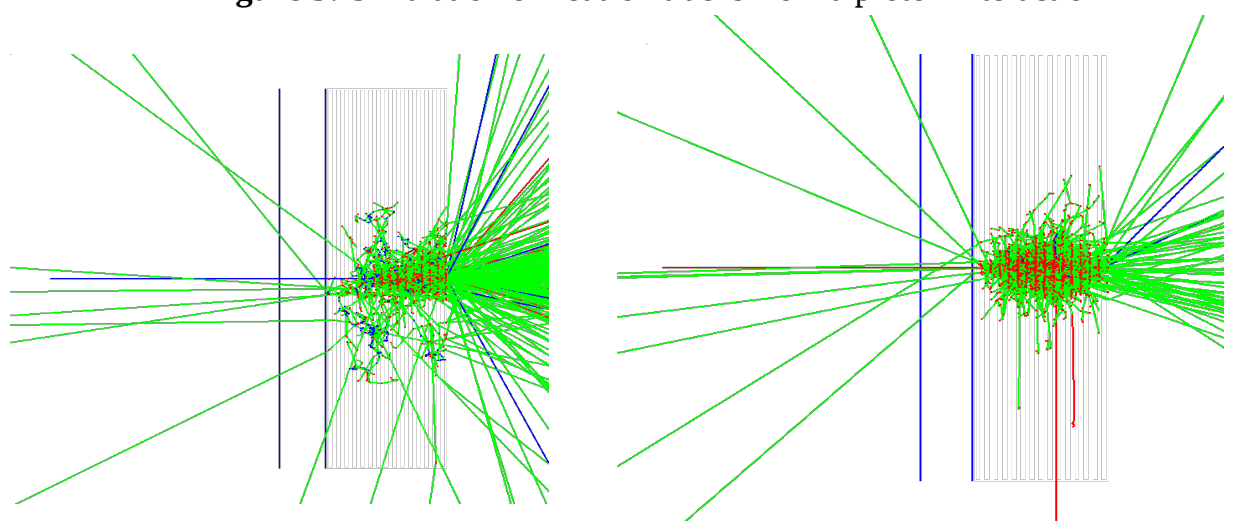

Figure 6: Examples of proton and electron events 


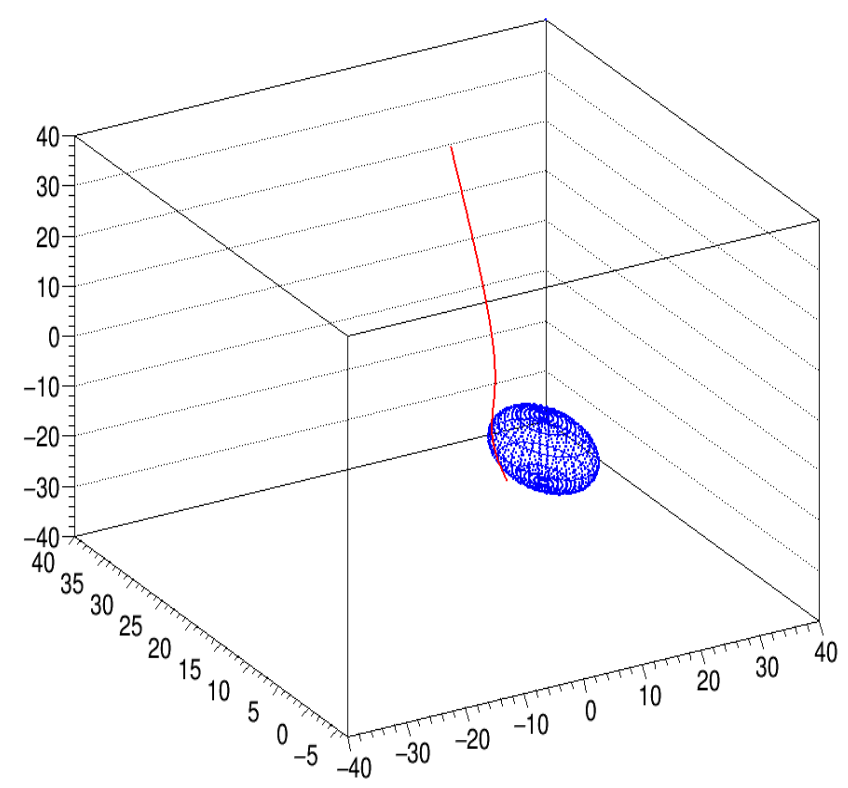

Figure 7: Simulstion of positron trajectory in the Earth magnetosphere (IGRF-12 model is used)
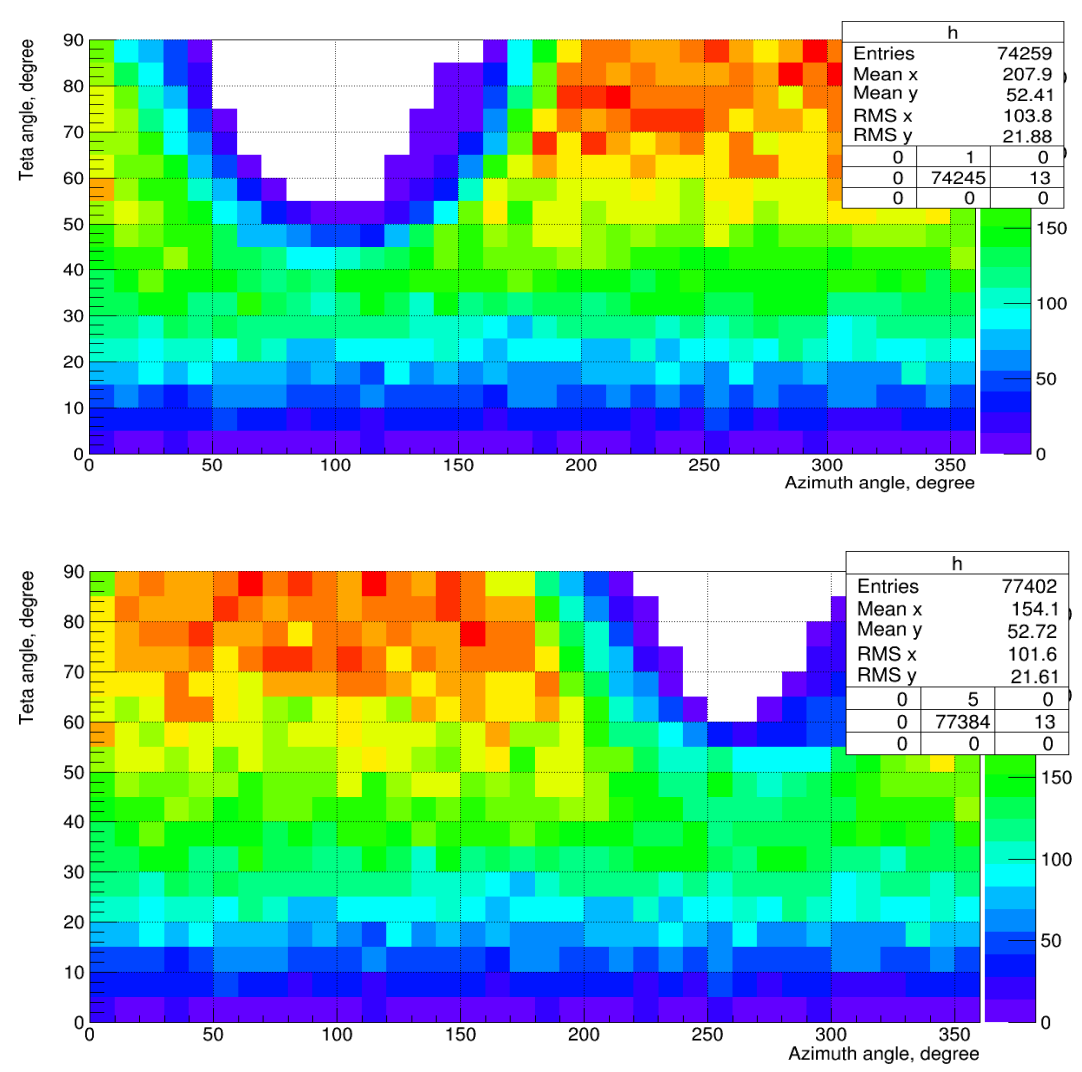

Figure 8: Simulated angular distribution of electrons (up) and positrons (down) with energy $20 \mathrm{GeV}$ in the instrument reference frame 


\section{Expected characteristic of the HERO}

Effective geometrical factor: protons $9 \mathrm{~m}^{2} \mathrm{sr}$; heavy nuclei $14 \mathrm{~m}^{2} \mathrm{sr}$; electrons and gamma $16 \mathrm{~m}^{2} \mathrm{sr}$.

Exposition factor (10 years flight): protons $90 \mathrm{~m}^{2} \mathrm{sr}$ year; heavy nuclei $140 \mathrm{~m}^{2} \mathrm{sr}$ year; electrons and gamma $160 \mathrm{~m}^{2} \mathrm{sr}$ year.

Energy resolution: protons $25 \%$, nuclei $\sim 10-15 \%$, electrons and gamma $\sim 5 \%$.

Charge resolution: 0.2 charge unit.

Rejection level: protons/electrons $10^{5}-10^{6}$, gamma/electrons $>10^{3}$.

Total mass 12 ton.

Power consumption $<3500 \mathrm{~W}$.

Overall dimensions: In transport situation the circumference is less than $2.5 \mathrm{~m}$ and the height is also less than $2.5 \mathrm{~m}$.

\section{Summary}

Summary The HERO project is presented. The equipment is intended for the solution of the most actual problems of high energy cosmic ray physics. The combination of the last achievements of physical experimental techniques and modern level of development of the space industry allows to create unique space complex which could carry out the break data in the space science.

\section{Acknowlegments}

We acknowledge support from Russian Academy Of Sciences and State Space Corporation ROSCOSMOS.

\section{References}

[1] H.S. Ahn, E.S. Seo, J.H. Adams et al., Adv. Space Res. 37(2006) 1950

[2] P. Boyle, D. Muller, M. Ave et al., Adv. Space Res. 42(2008) 409.

[3] E.S. Seo, Astropart. Phys. 39-40(2012) 76.

[4] O. Adriani, G. C. Barbarino, G. A. Bazilevskaya et al., Phys. Rev. Lett. 106(2011) 201101

[5] B.Bertucci, Proc.33rd ICRC (2013)1267. Rio-de-Janeiro. Brazilia.

[6] J. Chang, J. H. Adams, H. S. Ahn et al., Nature 456(2008) 362.

[7] M. Aguilar, G. Alberti, G.; B. Alpat et al., Phys. Rev. Lett. 110, 141102 (2013)

[8] O. Vasilyev, D. Karmanov, I. Kovalyov et al., Physics of Atomic Nuclei. 77(2014) 587

[9] P. Maestro, Journal of Physics: Conference Series 409(2013) 012026

[10]F.Gargano, arXiv:1701.05046 [astro-ph.HE] (2017) 